\title{
The evaluation of lymph node metastasis by endobronchial ultrasound-guided transbronchial needle aspiration: Crucial for selection of surgical candidates with metastatic lung tumors
}

Takahiro Nakajima, MD, ${ }^{a}$ Kazuhiro Yasufuku, MD, PhD, FCCP, ${ }^{a}$ Akira Iyoda, MD, PhD, ${ }^{\text {a }}$ Shigetoshi Yoshida, MD, PhD, Makoto Suzuki, MD, PhD, ${ }^{a}$ Yasuo Sekine, MD, PhD, ${ }^{a}$ Kiyoshi Shibuya, MD, PhD, ${ }^{a}$ Kenzo Hiroshima, MD, PhD, Yukio Nakatani, MD, PhD, and Takehiko Fujisawa, MD, $\mathrm{PhD}^{\mathrm{a}}$

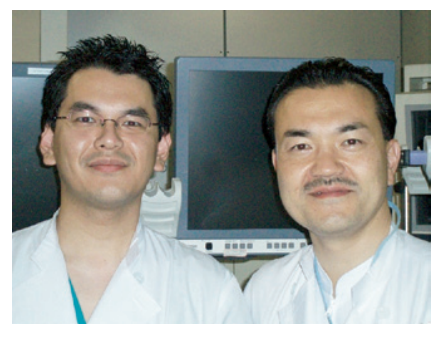

Drs Nakajima and Yasufuku (left to right)
From the Departments of Thoracic Surgery $^{\mathrm{a}}$ and Diagnostic Pathology, ${ }^{\mathrm{b}}$ Graduate School of Medicine, Chiba University, Chiba, Japan.

This study was supported in part by Grantsin-Aid for Scientific Research from the Ministry of Education, Culture, Sports, Science and Technology of Japan (T.N.; No. 18790986) and was also supported by The Emphasis Research Project by expenditure at the discretion of the president of Chiba University in 2006 (T.N.).

All authors read and approved the final manuscript.

Received for publication Jan 29, 2007; revisions received July 19, 2007; accepted for publication July 26, 2007.

Address for reprints: Kazuhiro Yasufuku, $\mathrm{MD}, \mathrm{PhD}$, Department of Thoracic Surgery, Graduate School of Medicine, Chiba University, 1-8-1 Inohana, Chuo-ku, Chiba, 2608670, Japan (E-mail: kyasufuku@faculty. chiba-u.jp).

J Thorac Cardiovasc Surg 2007;134:1485-90 $0022-5223 / \$ 32.00$

Copyright (C) 2007 by The American Association for Thoracic Surgery

doi:10.1016/j.jtcvs.2007.07.022
Objectives: Pulmonary metastasectomy is accepted as an effective treatment for properly selected patients with metastatic lung tumors. In such patients, the presence of mediastinal and/or hilar lymph node metastasis is a significant negative prognostic factor. The purpose of this study was to evaluate the usefulness of endobronchial ultrasound-guided transbronchial needle aspiration (EBUS-TBNA) for the detection of mediastinal and hilar lymph node metastasis in patients with potentially resectable metastatic lung tumors.

Methods: Patients with metastatic lung tumors with radiologically defined mediastinal and/or hilar lymph nodes on chest computed tomographic scans referred to our department for pulmonary resection were retrospectively analyzed. Successful lymph node aspiration was evidenced by the presence of malignant cells or normal lymphocytes. Cytologic and histologic analysis was used to confirm metastasis in surgically resected specimens unless metastasis was proven by EBUS-TBNA.

Results: A total of 106 patients were referred for metastasectomy during the study period. EBUS-TBNA was performed in 60 lymph nodes $(37$ mediastinal and 23 hilar nodes) from 43 patients. Cytologic and/or histologic samples were diagnostic in $41(95.3 \%)$ patients. EBUS-TBNA detected lymph node metastasis in 23 patients. The sensitivity, specificity, and diagnostic accuracy rate of EBUS-TBNA for diagnosis of mediastinal and hilar lymph node metastasis were $92.0 \%, 100 \%$, and $95.3 \%$, respectively.

Conclusions: EBUS-TBNA is a highly sensitive modality for the evaluation of mediastinal and hilar lymph node metastasis in patients with metastatic lung tumors. EBUS-TBNA allows preoperative histologic as well as cytologic evaluation of mediastinal and hilar lymph nodes.

$\mathrm{S}$ ince Barney and Churchill ${ }^{1}$ reported the long-term survival of a patient with metastatic lung tumor in the kidney in 1939, pulmonary metastasectomy has been widely adopted and surgical resection of pulmonary metastasis is considered a standard therapeutic procedure in properly selected patients. Removal of metastatic lung tumors from extrapulmonary neoplasms has been shown to improve patient survival. ${ }^{2-6}$ However, clinical evidence indicates that patients with mediastinal and/or hilar lymph node metastasis have a poor prognosis. ${ }^{7-9}$ Therefore, accurate mediastinal and hilar lymph node staging is critical for selecting patients suitable for metastasectomy. Although mediastinoscopy remains the gold standard for evaluation of the mediastinum, this procedure requires general anesthesia and is associated with certain complications, such as bleeding, recurrent nerve damage, 


\section{Abbreviations and Acronyms \\ CT $=$ computed tomography \\ EBUS-TBNA $=$ endobronchial ultrasound-guided \\ transbronchial needle aspiration}

and thoracic duct injury. Herein, we describe the use of endobronchial ultrasound-guided transbronchial needle aspiration (EBUS-TBNA), a minimally invasive procedure performed with the patient under local anesthesia, which enables mediastinal and hilar lymph node assessment with high sensitivity in patients with metastatic lung tumors from extrapulmonary neoplasms. ${ }^{10-15}$

\section{Patients and Methods \\ Patients}

We performed a retrospective chart review of patients with suspected or pathologically established metastatic lung tumors referred to the Department of Thoracic Surgery, Chiba University Hospital, from June 2002 to December 2006. Chest computed tomography (CT) was performed with single-injection contrast and multidetector-row CT (Light Speed; GE Medical System, Milwaukee, Wis). EBUS-TBNA was performed in patients with radiologically defined mediastinal and/or hilar lymph nodes with a short axis of $5 \mathrm{~mm}$ or more on enhanced chest CT. A total of 106 patients with proven or suspected metastatic lung tumors were referred for metastasectomy during the study period. Forty-three patients fulfilled these criteria and were included and 63 patients were excluded owing to the cutoff value of the lymph node size in this study. In all patients, the diagnosis of metastatic lung tumor from extrapulmonary lesion was established by preoperative biopsy or by final pathologic diagnosis of resected specimens. Patients with primary lung cancer were excluded from the study. Lymph node stations and numbers were determined according to the Japan Lung Cancer Society Classification. ${ }^{16}$ Written informed consent was obtained from all patients included in this study.

\section{EBUS-TBNA}

EBUS-TBNA was performed on an outpatient basis in patients under conscious sedation. Local anesthesia was achieved with 5 $\mathrm{mL}$ nebulized $1 \%$ lidocaine solution in the pharynx. A 2-mL bolus dose of $2 \%$ lidocaine was also used during the procedure. The bronchoscope was inserted orally during midazolam-induced conscious sedation. Patients underwent electrocardiography, pulse oximetry, and blood pressure monitoring without the presence of an anesthesiologist. Convex probe EBUS (BF-UC260F-OL8; Olympus, Tokyo, Japan) was used to examine mediastinal and hilar lymph nodes. The convex probe EBUS is integrated with a convex transducer $(7.5 \mathrm{MHz})$ that scans parallel to the insertion direction of the bronchoscope. Images can be obtained by directly contacting the probe or by attaching a balloon on the tip and inflating with saline. The ultrasound image is processed in a dedicated ultrasound scanner (EU-C2000; Olympus) and is visualized along with the conventional bronchoscopy image on the same monitor. A dedicated 22-gauge needle was used to perform transbronchial needle aspiration (NA-201SX-4022; Olympus). The inner diameter of this needle is nearly equal to that of conventional 21-gauge needles, which allows sampling of histologic cores. The needle is also equipped with an internal sheath that is withdrawn after passing the bronchial wall, avoiding contamination during EBUS-TBNA. The needle can be visualized through the optic device and on the ultrasound image.

\section{Lymph Node Sampling}

The dedicated 22-gauge needle was used for lymph node sampling. After the initial puncture, the internal sheath was used to clean out the internal lumen, which becomes clogged with bronchial membrane. The internal sheath is then removed and negative pressure is applied with a syringe. After the needle is moved back and forth inside the lymph node, the needle is retrieved and the internal sheath is used once again to push out the histologic core. ${ }^{11}$ With this method, histologic cores as well as cytologic specimens can be obtained. The aspirated material was smeared onto glass slides, and smears were air-dried and immediately stained with Diff-Quik (Sysmex Corporation, Kobe, Japan) for immediate interpretation by an on-site cytopathologist to confirm adequate cell material. Furthermore, Papanicolaou staining and light microscopy were performed by an independent cytopathologist who was blinded to the details of patient information. Histologic cores were fixed with formalin and stained with hematoxylin and eosin. Immunohistochemistry was also performed in some patients.

EBUS-TBNA diagnosis was confirmed by open thoracotomy, thoracoscopy, or clinical follow-up. In patients with malignant lymph nodes, the determination was based on malignant cytologic and/or histologic results at EBUS-TBNA or surgical-pathologic confirmation. In patients with benign lymph nodes, this determination was based on surgical pathologic confirmation of EBUSTBNA-targeted nodes by lymph node dissection of the lymph node station of interest or results of clinical follow-up for at least 6 months demonstrating a lack of clinical or radiologic disease progression.

\section{Data Analysis}

The sensitivity, specificity, positive predictive value, negative predictive value, and diagnostic accuracy rate were calculated by standard definitions. This study was approved by the ethical committee at our institute and a written consent form was obtained from all the patients who participated in the study.

\section{Results}

\section{Patient Characteristics}

Characteristics of the 43 patients who participated in this study are listed in Table 1 . Sixty lymph nodes (37 mediastinal and 23 hilar lymph nodes) were evaluated. There were 27 men and 16 women, and the average age was 63.3 years. The primary malignancy sites are listed in Table 1.

\section{EBUS-TBNA Results}

The subcarinal lymph node (\#7) and interlobar lymph node (\#11) were the most frequently punctured lymph node stations (Table 2). Lymph node stations assessed by EBUSTBNA are shown in Table 2. The short axes of the lymph 
TABLE 1. Patient characteristics

\begin{tabular}{lcc}
\hline Age (y) & & \\
Mean & 63.3 & \\
Range & $31-83$ & \\
Gender (n = 43) & & \\
Male & 27 & \\
Female & 16 & Metastatic rate \\
Primary tumor type & & $9 / 16(56.3 \%)$ \\
Colorectal cancer & 16 & $2 / 8(25 \%)^{*}$ \\
Head and neck cancer & 8 & $4 / 6(66.7 \%)$ \\
Breast cancer & 6 & $4 / 5(80 \%)$ \\
Renal cell carcinoma & 5 & $0 / 2(0 \%)$ \\
Esophageal cancer & 2 & $1 / 1(100 \%)$ \\
Ovarian cancer & 1 & $1 / 1(100 \%)$ \\
Hepatocellular carcinoma & 1 & $1 / 1(100 \%)$ \\
Prostate cancer & 1 & $0 / 1(0 \%)$ \\
Germ cell tumor & 1 & \\
Malignant melanoma & 1 & \\
Eccrine carcinoma & 1 & \\
No. of metastatic lesions & & \\
Solitary & 32 & \\
Multiple (2-4 lesions) & 11 & \\
Mean & 1.42 & \\
Disease-free interval & & \\
Range (mo) & 44.27 & \\
Mean (mo) & & \\
\hline
\end{tabular}

*Two patients in whom endobronchial ultrasound-guided transbronchial needle aspiration was not diagnostic.

nodes based on chest CT and EBUS ranged from 5 to 35 $\mathrm{mm}$ and 3.9 to $28.8 \mathrm{~mm}$, respectively (Table 2 ). The relationship between lymph node size on CT scan and likelihood of metastasis is described in Table 3. Four (36.4\%) of 11 lymph nodes with a short axis of $5 \mathrm{~mm} \leq$ lymph node $<$ $10 \mathrm{~mm}$ on $\mathrm{CT}$ were positive for metastasis. In addition, 9

TABLE 2. Lymph node stations assessed by EBUS-TBNA

\begin{tabular}{ccc}
\hline & $\begin{array}{c}\text { CT, mean size } \\
\text { in mm (range) }\end{array}$ & $\begin{array}{c}\text { EBUS, mean size } \\
\text { in mm (range) }\end{array}$ \\
\hline Station & & \\
$\# 1(\mathrm{n}=1)$ & 20 & 20.0 \\
$\# 3(\mathrm{n}=15)$ & $12(5-28)$ & $10.5(3.9-21.4)$ \\
$\# 3 \mathrm{p}(\mathrm{n}=2)$ & $10(10-10)$ & $11.2(8.2-14.2)$ \\
$\# 4(\mathrm{n}=2)$ & $7(5-9)$ & $7.3(6.3-8.3)$ \\
$\# 7(\mathrm{n}=17)$ & $11(5-21)$ & $13.9(7.9-21.7)$ \\
$\# 10(\mathrm{n}=5)$ & $13(10-16)$ & $16.3(10.9-20.2)$ \\
$\# 11(\mathrm{n}=17)$ & $16(8-35)$ & $14.9(7.6-28.8)$ \\
$\# 12(\mathrm{n}=1)$ & 14 & 14.4 \\
Regional & & \\
Mediastinum $(\mathrm{n}=37)$ & & $13.6(3.9-28.8)$ \\
Hilar or lobar (n=23) & &
\end{tabular}

$\overline{E B U S-T B N A, \text { Endobronchial ultrasound-guided transbronchial needle as- }}$ piration; $C T$, computed tomography.
TABLE 3. Association between lymph node size on CT scan and likelihood of metastasis

\begin{tabular}{lrcc}
\hline Short axis on CT & $\mathbf{n}$ & $\begin{array}{c}\text { Negative for } \\
\text { metastasis }\end{array}$ & $\begin{array}{c}\text { Positive for } \\
\text { metastasis }\end{array}$ \\
\hline $5 \mathrm{~mm} \leq \mathrm{LN}<10 \mathrm{~mm}$ & 11 & 7 & 4 \\
$10 \mathrm{~mm} \leq \mathrm{LN}<15 \mathrm{~mm}$ & 16 & 11 & 5 \\
$15 \mathrm{~mm} \leq \mathrm{LN}<20 \mathrm{~mm}$ & 6 & 2 & 4 \\
$20 \mathrm{~mm} \leq \mathrm{LN}$ & 10 & 0 & 10 \\
Mean (n $=43)$ & & $9.53 \mathrm{~mm}$ & $16.75 \mathrm{~mm}$
\end{tabular}

$C T$, Computed tomography; $L N$, lymph node.

(33.3\%) of 27 lymph nodes were positive for metastasis even for nodes less than $15 \mathrm{~mm}$ on CT (Table 3). Pathologic diagnosis was achieved in $41(95.3 \%)$ of 43 patients, and lymph node metastases were diagnosed in 23 patients. In 2 patients, specimens obtained by EBUS-TBNA were inadequate for pathologic evaluation (no lymphocytes in the sample). These 2 patients had metastasis in the final resected specimen, and therefore their results were considered false negative. The sensitivity, specificity, diagnostic accuracy rate, positive predictive value, and negative predictive value of EBUS-TBNA for diagnosis of mediastinal and hilar lymph node metastasis were $92.0 \%, 100 \%, 95.3 \%, 100 \%$, and $90 \%$, respectively (Table 4). Histologic cores were obtained in $28(65.1 \%)$ of the 43 patients. In all 23 patients, lymph node metastasis was diagnosed by pathologic examination (in 7 patients by cytologic examination alone, in 14 patients by both cytologic and histologic examination, and in 2 patients by histologic examination alone). Immunohistochemistry was additionally performed in 7 patients ( 2 with colorectal cancer, 1 with renal cell carcinoma, 1 with prostate cancer, 1 with melanoma, 1 with thyroid cancer, and 1 with ovarian cancer) (Figures 1 and 2).

Twenty of 23 patients with lymph node metastases received chemotherapy or immunotherapy. No patients had any complications related to EBUS-TBNA.

\section{Discussion}

This is the first study to evaluate the efficacy of EBUSTBNA in the preoperative detection of mediastinal and/or

TABLE 4. Comparison of EBUS-TBNA results of lymph nodes with final diagnosis

\begin{tabular}{lccc}
\hline & \multicolumn{3}{c}{ EBUS-TBNA results } \\
\cline { 2 - 4 } Final diagnosis & Malignant & Benign & Total \\
\hline Malignant & 23 & $2^{*}$ & 25 \\
Benign & 0 & 18 & 18 \\
Total & 23 & 20 & 43 \\
\hline
\end{tabular}

EBUS-TBNA, Endobronchial ultrasound-guided transbronchial needle aspiration. The sensitivity, specificity, and diagnostic accuracy rate of EBUSTBNA for diagnosis of mediastinal and hilar lymph node metastasis were $92.0 \%, 100 \%$, and $95.3 \%$, respectively. ${ }^{*}$ Inadequate specimen. 

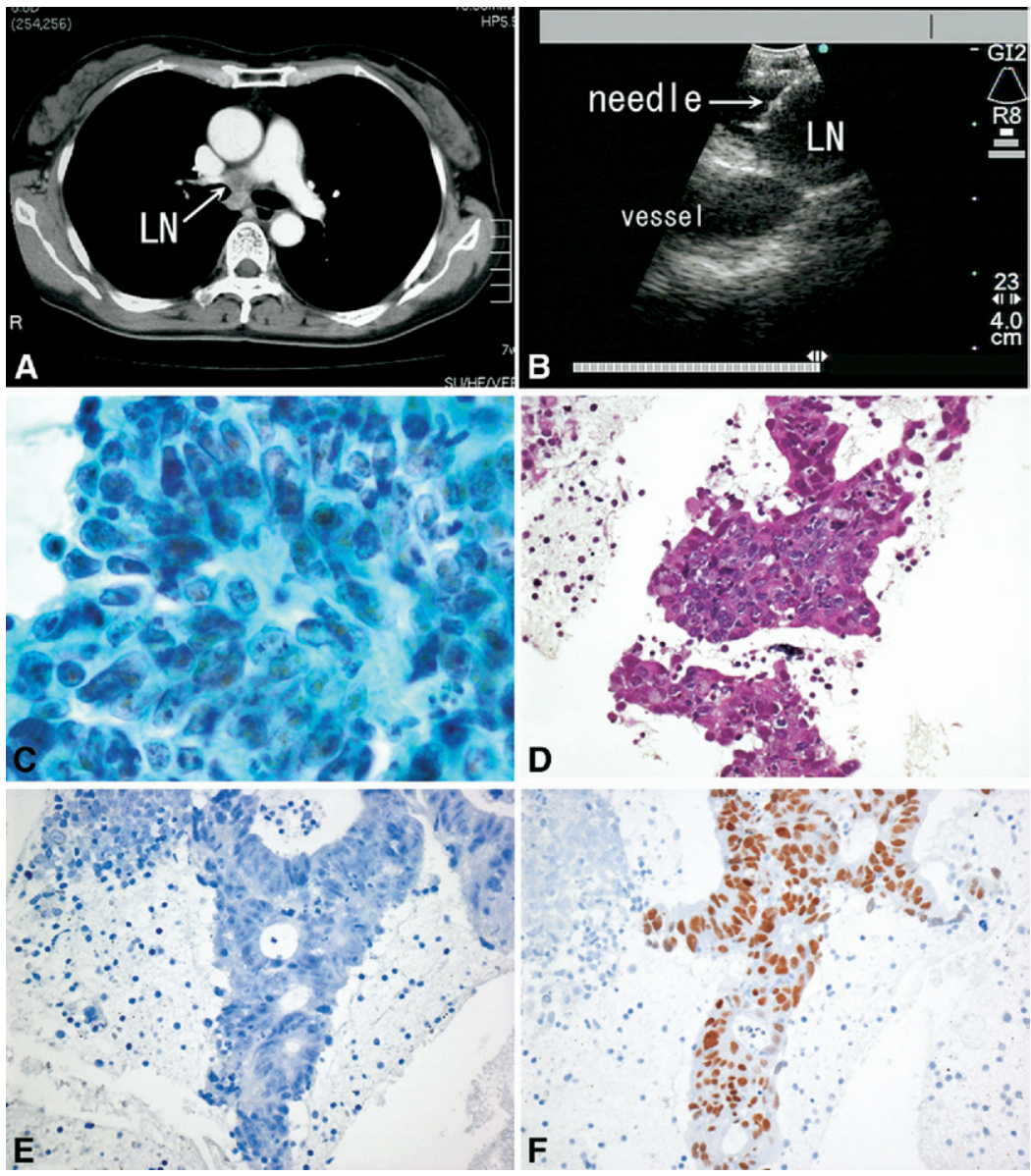

Figure 1. Chest CT showed subcarinal lymph node enlargement (A). EBUS-TBNA was performed for the enlarged subcarinal lymph node (B). Cytologic findings showed atypical columnar cells with Papanicolaou stain (C). Histologic findings revealed adenocarcinoma with palisading proliferation (D). Immunohistochemical analysis revealed that tumor cells were negative for thyroid transcription factor-1 (E) and positive (brown stain) for CDX2 (F). LN, Lymph node.

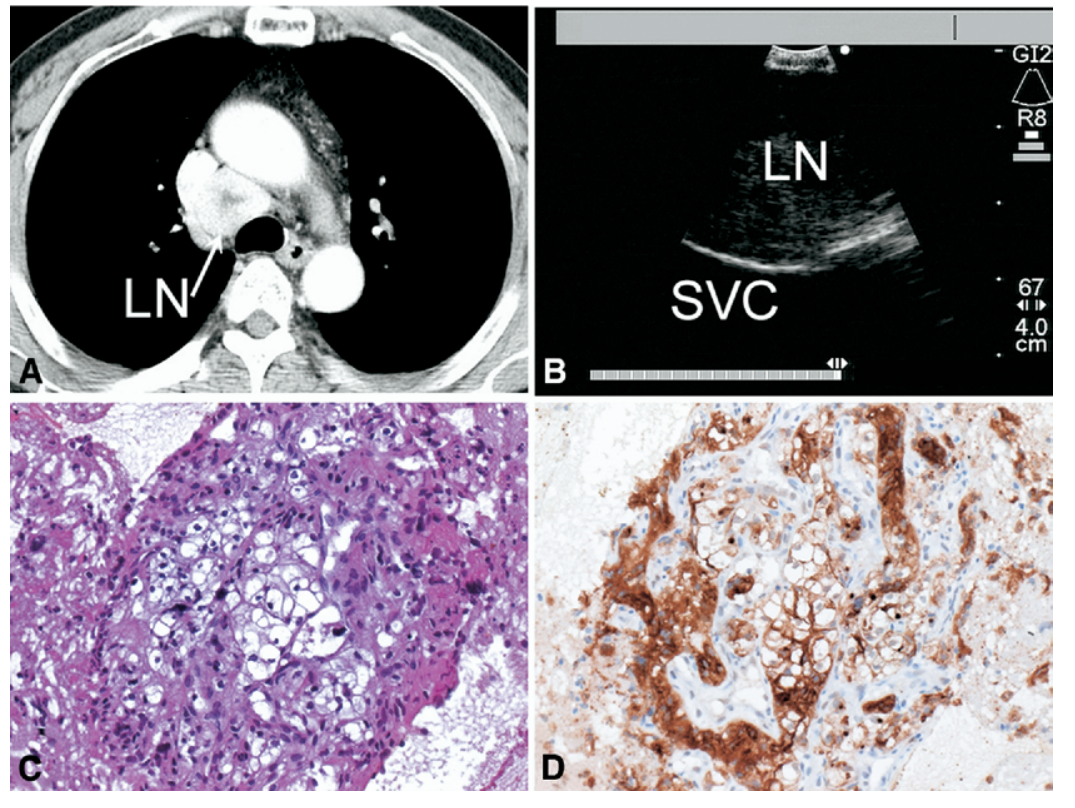

Figure 2. Chest CT showed an enhanced pretracheal lymph node enlargement (A). Convex probe EBUS showed the enlarged pretracheal lymph node (B). Histologic findings showed tumor cells with clear cytoplasm arranged in nests separated by fibrous septa (C). Immunohistochemical analysis showed CD10-positive epithelial tumor cells (D). LN, Lymph node; SVC, superior vena cava. 
hilar lymph node metastasis in patients with metastatic lung tumor from extrapulmonary lesions. From our experience in patients with lung cancer, lymph nodes measuring $5 \mathrm{~mm}$ or larger can clearly be detected by EBUS and can be sampled by EBUS-TBNA. Hence, we considered patients with mediastinal and/or hilar lymph nodes with a short axis of $5 \mathrm{~mm}$ or more to be optimal candidates for EBUS-TBNA. From the results of this study, $34.6 \%$ of the lymph nodes less than $10 \mathrm{~mm}$ in size on chest CT showed lymph node metastasis and 9 of 27 lymph nodes were positive for metastasis even for nodes less than $15 \mathrm{~mm}$.

Pulmonary metastasectomy is widely accepted as an effective treatment for properly selected patients with different tumor types. ${ }^{2-6}$ Some investigators have reported that patients with mediastinal or hilar lymph node metastasis have a poor prognosis..$^{7-9}$ Although the presence of mediastinal and/or hilar lymph node metastasis is one of the most important factors for selecting patients who will benefit from pulmonary metastasectomy, few reports have discussed preoperative evaluation of metastases in these nodes. ${ }^{17}$ After a retrospective chart review, Ercan and associates ${ }^{7}$ reported that $20(28.6 \%)$ of 70 patients with metastatic lung tumors who underwent complete lymph node dissection had mediastinal and/or hilar lymph node metastasis. In other reports, the rate of mediastinal and/or hilar lymph node metastasis has ranged from $8.6 \%$ to $14.3 \%{ }^{7}$ The metastatic rate in these nodes in our current study $(56.1 \%)$ is higher than those in previous reports owing to the design of the current study - in this study, patients with lymph nodes measuring less than $5 \mathrm{~mm}$ on CT were excluded. However, our study shows that almost half of the patients who have radiologically detectable lymph nodes have lymph node metastasis proven by EBUS-TBNA. The overall prevalence of patients with lymph node metastasis in our series was $21.7 \%$ (23/106 patients) and is similar to the prevalence described in previous reports. ${ }^{7}$

New diagnostic modalities such as multidetector helical $\mathrm{CT}$, positron emission tomography, and positron emission tomography combined with CT have been reported to be useful for the detection of mediastinal and hilar lymph node metastases in patients with non-small cell lung cancer. ${ }^{18-20}$ However, the diagnostic accuracy of these imaging modalities is limited because they do not allow pathologic confirmation. Mediastinoscopy remains the standard procedure for diagnosis of mediastinal lymph node metastasis. Murthy and colleagues ${ }^{17}$ reported the importance of preoperative determination of resectability for metastasectomy from renal cell carcinoma and recommended that mediastinoscopy is a valuable tool for optimizing patient selection. However, mediastinoscopy is invasive, requires general anesthesia, and does not allow evaluation of hilar lymph nodes.

$\mathrm{We}^{10-12}$ have reported the utility of EBUS-TBNA for evaluation of the mediastinum in lung cancer. EBUS-TBNA permits investigation of the majority of the mediastinum; in addition, isolation of tissue samples can be obtained for histologic diagnosis, including immunohistochemistry. ${ }^{10-15}$ Immunohistochemistry has a great impact on the diagnosis of metastasis from extrapulmonary lesions, because it provides additional information concerning tumor origin. ${ }^{21}$ Furthermore, recent advances in biomarker research enable the use of immunohistochemistry to predict response to systemic therapy for certain tumors. ${ }^{22,23}$ This raises the possibility of "tailor-made treatment," in which patients with metastatic lung tumor with lymph node involvement may receive different types of treatment based on the biological nature of the tumor at the metastatic site. In addition, EBUS-TBNA is a minimally invasive procedure that can be performed in an outpatient setting; none of our patients has ever had complications related to this procedure.

In conclusion, preoperative evaluation of mediastinal and hilar lymph nodes can be performed by EBUS-TBNA in patients with metastatic lung tumors. EBUS-TBNA allows cytologic as well as histologic evaluation of the mediastinum and the hilum.

We thank Ms Fumie Saegusa and Mr Fumio Horiuchi for support of cytologic diagnosis.

\section{References}

1. Barney JD, Churchill EJ. Adenocarcinoma of the kidney with metastasis to the lung cured by nephrectomy and lobectomy. J Urol. 1939; 42:269-74.

2. Kondo H, Okumura T, Ohde Y, Nakagawa K. Surgical treatment for metastatic malignancies. Pulmonary metastasis: indications and outcomes. Int J Clin Oncol. 2005;10:81-5.

3. Monteiro A, Arce N, Bernardo J, Eugenio L, Antunes MJ. Surgical resection of lung metastases from epithelial tumors. Ann Thorac Surg. 2004;77:431-7.

4. Kandioler D, Kromer E, Tuchler H, End A, Muller MR, Wolner E, et al. Long-term results after repeated surgical removal of pulmonary metastases. Ann Thorac Surg. 1998;65:909-12.

5. Todd TR. The surgical treatment of pulmonary metastases. Chest. 1997; 112:287S-90S.

6. The International Registry of Lung Metastases Writing Committee: Pastorino U, Buyse M, Friedel G, Ginsberg RJ, Girard P, Goldstraw $\mathrm{P}$, et al.Long-term results of lung metastasectomy: prognostic analyses based on 5206 cases. J Thorac Cardiovasc Surg. 1997;113: 37-49.

7. Ercan S, Nichols FC, Trastek VF, Deschamps C, Allen MS, Miller DL, et al. Prognostic significance of lymph node metastasis found during pulmonary metastasectomy for extrapulmonary carcinoma. Ann Thorac Surg. 2004;77:1786-91.

8. Pfannschmidt J, Muley T, Hoffmann H, Dienemann H. Prognostic factors and survival after complete resection of pulmonary metastases from colorectal carcinoma: experiences in 167 patients. $J$ Thorac Cardiovasc Surg. 2003;126:732-9.

9. Iizasa T, Suzuki M, Yoshida S, Motohashi S, Yasufuku K, Iyoda A, et al. Prediction of prognosis and surgical indications for pulmonary metastasectomy from colorectal cancer. Ann Thorac Surg. 2006;82: 254-60.

10. Yasufuku K, Chiyo M, Sekine Y, Chhajed PN, Shibuya K, Iizasa T, et al. Real-time endobronchial ultrasound-guided transbronchial needle aspiration of mediastinal and hilar lymph nodes. Chest. 2004;126: $122-8$. 
11. Yasufuku K, Chiyo M, Koh E, Moriya Y, Iyoda A, Sekine Y, et al. Endobronchial ultrasound guided transbronchial needle aspiration for staging of lung cancer. Lung Cancer. 2005;50:347-54.

12. Yasufuku K, Nakajima T, Motoori K, Sekine Y, Shibuya K, Hiroshima $\mathrm{K}$, et al. Comparison of endobronchial ultrasound, positron emission tomography, and CT for lymph node staging of lung cancer. Chest. 2006;130:710-8.

13. Rintoul RC, Skwarski KM, Murchison JT, Wallace WA, Walker WS, Penman ID. Endobronchial and endoscopic ultrasound-guided realtime fine-needle aspiration for mediastinal staging. Eur Respir J. 2005;25:416-21.

14. Herth FJ, Ernst A, Eberhardt R, Vilmann P, Dienemann H, Krasnik M. Endobronchial ultrasound-guided transbronchial needle aspiration of lymph nodes in the radiologically normal mediastinum. Eur Respir J. 2006;28:910-4.

15. Herth FJ, Eberhardt R, Vilmann P, Krasnik M, Ernst A. Real-time endobronchial ultrasound guided transbronchial needle aspiration for sampling mediastinal lymph nodes. Thorax. 2006;61:795-8.

16. The Japan Lung Cancer Society. Part I. Anatomy: The Japan Lung Cancer Society. Classification of lung cancer. 1st English ed. Tokyo: Kanehara and Co., Ltd; 2000. p. 1-14.

17. Murthy SC, Kim K, Rice TW, Rajeswaran J, Bukowski R, DeCamp $\mathrm{MM}$, et al. Can we predict long-term survival after pulmonary metastasectomy for renal cell carcinoma? Ann Thorac Surg. 2005;79:9961003 .
18. Pozo-Rodriguez F, Martin de Nicolas JL, Sanchez-Nistal MA, Maldonado A, Garcia de Barajas S, Calero-Garcia R, et al. Accuracy of helical computed tomography and $[18 \mathrm{~F}]$ fluorodeoxyglucose positron emission tomography for identifying lymph node mediastinal metastases in potentially resectable non-small-cell lung cancer. J Clin Oncol. 2005;23:8348-56.

19. Gould MK, Kuschner WG, Rydzak CE, Maclean CC, Demas AN, Shigemitsu $\mathrm{H}$, et al. Test performance of positron emission tomography and computed tomography for mediastinal staging in patients with non-small-cell lung cancer: a meta-analysis. Ann Intern Med. 2003; 139:879-92.

20. Halpern BS, Schiepers C, Weber WA, Crawford TL, Fueger BJ, Phelps ME, et al. Presurgical staging of non-small cell lung cancer: positron emission tomography, integrated positron emission tomography/CT, and software image fusion. Chest. 2005;128:2289-97.

21. Nakajima T, Yasufuku K, Wong M, Iyoda A, Suzuki M, Sekine Y, et al. Histological diagnosis of mediastinal lymph node metastasis from renal cell carcinoma by endobronchial ultrasound guided transbronchial needle aspiration: a case report. Respirology. 2007;12:302-3.

22. Ross JS, Fletcher JA. The HER-2/neu oncogene in breast cancer: prognostic factor, predictive factor, and target for therapy. Stem Cells. 1998;16:413-28.

23. Adlard JW, Richman SD, Seymour MT, Quirke P. Prediction of the response of colorectal cancer to systemic therapy. Lancet Oncol. 2002;3:75-82. 